Cahiers $d u$ MONDE RUSSE

\section{Cahiers du monde russe}

Russie - Empire russe - Union soviétique et États indépendants

$50 / 2-3 \mid 2009$

L'Europe orientale, 1650-1730. Crises, conflits et renouveau

\title{
From Poets to PadonkiIngunn LUNDE
}

, Bergen : University of Bergen, 2009, 349 p. (Slavica Bergensia, 9)

\section{Jean Breuillard}

\section{(2) OpenEdition}

\section{Journals}

Édition électronique

URL : https://journals.openedition.org/monderusse/9780

DOI : $10.4000 /$ monderusse.9780

ISSN : $1777-5388$

Éditeur

Éditions de l'EHESS

Édition imprimée

Date de publication : 15 septembre 2009

ISBN : 978-2-7132-2260-3

ISSN : $1252-6576$

Référence électronique

Jean Breuillard, "From Poets to Padonkilngunn LUNDE », Cahiers du monde russe [En ligne], 50/2-3 |

2009, mis en ligne le 14 janvier 2013, consulté le 03 septembre 2022. URL : http://

journals.openedition.org/monderusse/9780; DOI : https://doi.org/10.4000/monderusse.9780

Ce document a été généré automatiquement le 3 septembre 2022

Tous droits réservés 


\title{
From Poets to Padonkilngunn LUNDE
}

\author{
, Bergen : University of Bergen, 2009, 349 p. (Slavica Bergensia, 9) \\ Jean Breuillard
}

\section{Ingunn LUNDE, Martin PAULSEN, eds., From Poets to Padonki, Linguistic Authority \& Norm Negotiation in Modern Russian Culture, Bergen : University of Bergen, 2009, 349 p. (Slavica Bergensia, 9)}

1 « Nihil est in lingua quod non prius fuerit in oratione.» Les changements dans la langue sont perçus au début comme des variations de l'usage et donc de la norme. Ingunn Lunde, professeur à l'université de Bergen, fondatrice et directrice de la série "Slavica Bergensia ", est l'une des slavistes occidentales les plus attentives aux changements que connaît le russe d'aujourd'hui et à l'évolution de la norme chez les russophones. Elle pilote, dans le cadre du Conseil de la recherche scientifique de Norvège, le projet " Landslide of the Norm: Linguistic liberalization and literary development in Russia in the 1920s and 1990s » dont on trouvera le descriptif sur le site http://www.hf.uib.no/i/ russisk/landslide/home.html. En collaboration avec Martin Paulsen, docteur de l'université de Bergen, Ingunn Lunde rassemble ici dix-sept contributions, toutes consacrées au rapport à la norme. Dix articles sont en anglais; les autres sont en russe.

Roman Jakobson avait qualifié le changement de norme après 1917 d'« éboulement » ou de "glissement de terrain" (Erdrutsch der Norm, landslide of the norm). Tynjanov y aperçut, quant à lui, ce qu'il appelait un « intervalle » (promežutok). Ce bouleversement accompagna l'époque du mouvement futuriste et de ses audaces linguistiques (Vl. Majakovskij, V. Hlebnikov, D. Burljuk). C'est ce terme de landslide que reprend Ingunn Lunde, en l'étendant aux bouleversements consécutifs à la chute du communisme soviétique, en 1991. À chaque fois, le déplacement de la norme a provoqué des réactions contraires. C'est vrai de l'apparition de la discipline appelée kul'tura reči [la culture du langage] qui apparaît sous Stalin (terme qui suppose que la langue s'entretient, se cultive, s'amende et nécessite l'action de spécialistes habilités à dire le bon usage : les 
grammairiens, les gardiens de l'usage, les « hommes habiles », comme on disait dans la France de Vaugelas). C'est toujours vrai aujourd'hui, où les provocations émanant du mouvement des Padonki [les Crades] (altération graphique délibérée du mot podonki [la lie de la société ; les déchets ; les trashes]) provoquent des crispations. On lira avec intérêt la solide étude de Heinrich Kirschbaum (université de Passau), qui retrace l'histoire de l'image ambivalente du "glissement de la norme », dans les textes des formalistes, avec un accent particulier mis sur Tynjanov et Jakobson (p. 194-211). C'est donc tout un siècle d'histoire de la langue russe qui est couvert dans ce recueil.

Dans sa définition des six fonctions du langage, Roman Jakobson isolait la fonction "métalinguistique », qui permet de parler du langage en utilisant le langage, qui est en l'occurrence à la fois objet et outil. L'option retenue dans le recueil est qu'on ne saurait confiner le domaine de la métalangue aux grammaires, aux dictionnaires et autres ouvrages normatifs. Henning Andersen (UCLA) développe sur ce thème l'idée de «living norms ", selon laquelle aucun énoncé n'est totalement pur de métalangue. Et ce n'est pas un hasard si son article ouvre le volume. Dire quelque chose, c'est toujours le dire à certains auditeurs, à un certain moment et dans un certain but; le locuteur, à chaque fois, « négocie » son rapport à la norme. Bref, la pragmatique du langage doit intégrer la négociation de la norme. Dès lors, l'étude de la norme s'étend à toutes les pratiques d'écriture, pourvu cependant que le locuteur ou le scripteur dispose d'une palette langagière suffisante pour " négocier ", justement. On ne négocie que si l'on a une marge de manœuvre, la capacité d'employer tel mot et pas tel autre. Le russophone cultivé qui écrit Preved! [éq. : Ça l'eût !] au lieu de Privet ! [Salut !] joue sur le registre de la complicité et de la connivence culturelle. Il n'en va pas de même pour le scripteur qui écrit le même mot sans soupçonner une seconde qu'il enfreint l'orthographe. Le premier fait un usage raffiné de la transgression, le second appartient à la catégorie que les sociolinguistes soviétiques appelaient drôlement un moč « maloobrazovannyj čelovek» [personne peu instruite]. Les blogs sur Internet et les commentaires laissés par les internautes offrent une moisson inépuisable aux observateurs de la norme; et cela est vrai de toutes les langues. La question, en définitive, est toujours la même: l'infraction à la norme académique est-elle délibérée ou n'est-elle que le reflet de l'ignorance? Dans le second cas, on ne peut parler de négociation. Y a-t-il vraiment négociation de la norme à chaque fois qu'il y a énoncé ? Là est sans doute le point délicat de la thèse générale qui charpente le recueil.

C'est à cette argumentation qu'Ingunn Lunde consacre son article, dont le titre même est explicite : «Performative Metalanguage: Negotiating Norms Through Verbal Action » (p. 110-128). Elle distingue les récents textes métalinguistiques (parmi lesquels l'essaipamphlet de Maksim Krongauz, Russkij jazyk na grani nervnogo sryva [La langue russe au bord de la crise de nerfs], 2007), et montre comment des phénomènes nouveaux touchant à la dérivation et à la néologie peuvent conduire à un changement de perception de la norme. On pourrait élargir sa réflexion en rappelant que la Russie a connu, dans son histoire, fort peu de moments où la langue a été l'objet d'une approche formelle et ludique; citons la trop courte influence de la poésie baroque au XVII siècle et les poèmes-dessins de Simeon de Polock. La Russie n'a pas connu l'expérience des contraintes poétiques, illustrée, en France, par la poésie des Grands Rhétoriqueurs; elle n'a pas eu davantage l'équivalent, à l'époque moderne, de l'OuLiPo ou des lipogrammes, sauf sous une forme fondamentalement grave et sérieuse, comme le travail des futuristes et de Hlebnikov, poète « futurien» (budetljanin), sur les signifiants. 
Martin Paulsen (université de Bergen) s'intéresse aux discussions sur la norme dans la vie littéraire postsoviétique (p. 34-48). On lira avec intérêt deux contributions sur la contreculture des Padonki ; en particulier celle de Gusejn Gusejnov (MGU), dont les travaux sur la culture Internet font autorité; il analyse ici les réactions des internautes provoquées par deux des néologismes qu'il a créés: liturativ et èrrativ; et celle de Vera Zvereva (Institut de littérature mondiale, Moscou), qui cite le Monefest Padonkaff [Manifeste des Crades], dont la proposition 3 stipule : « Fsem učetiljam ruskava jazyka i lejterotury škol i vuzov - vypet' jadu " [Tout lè profs de langue et littérature russes dé zécoles et des faks douavent à valer du pouazon] (p. 47-79). On vérifiera avec amusement que les règles et la norme renaissent toujours dès qu'on tente de les éradiquer : ainsi, un utilisateur, dans le rôle de l'éternel grammairien, explique ici doctement que les graphies aftar (pour avtor) [auteur], kniška (pour knižka) [livre] sont correctes en langage èrrativ, mais non $f$ gazetu, [tans le journal], fruku [tans la main]. Il y a donc bien une grammaire du padonkistajl [le style crade]. Elena Markasova (université de Saint-Pétersbourg) étudie l'emploi actuel des structures parenthétiques (p. 80-86). Ellen Rutten observe les blogs de certains écrivains contemporains, et plus particulièrement celui de Tat'jana Tolstaja, et constate que leur pratique d'écriture peut ne pas être affectée par l'emploi de ce nouveau média (p. 97-109). Tine Roesen (université de Aarhus) étudie le roman d'Aleksej Slapovskij, Oni [Eux], dans lequel le personnage tente de s'opposer au discours idéologique ambiant en créant son propre idiome (p. 129-142). Le romancier Vladimir Sorokin fait l'objet de deux études. Dirk Uffelmann (université de Passau) analyse son évolution par rapport à la norme littéraire, depuis le roman de jeunesse Norma [La Norme] jusqu'à son récent Den' opričnika [Journée d'un opričnik] (p. 143-167). Karin Grelz (université de Lund) [p. 168-183] s'aventure dans la confrontation de Tridcataja ljubov' Mariny [Le Trentième amour de Marina] de Sorokin avec Krysolov [Le Charmeur de rats] de Marina Cvetaeva, en tentant de montrer que le roman du premier est modelé par... le poème de la seconde. La chercheuse voit dans les deux œuvres de véritables manifestes par lesquels les auteurs signifient leur refus de négocier la norme, au nom de l'autonomie créatrice de l'artiste. Il faudrait toutefois se demander quelle norme enfreint au juste Sorokin: est-il si différent de Barkov, au XVIII ${ }^{\mathrm{e}}$ siècle, et de ses contemporains licencieux français? En quoi est-il subversif? Peter Alberg Jensen (université de Stockholm) dégage chez Boris Pasternak la même orgueilleuse revendication (p. 184-193). Pasternak, dans ses premiers essais, développait l'idée selon laquelle l'art s'accomplit aux dépens de la norme, au point que la réalité n'est véritablement atteinte que lorsque les choses sont libérées de leur nom. C'est également Pasternak qu'étudie Susanna Witt (université de Stockholm), qui interprète son concept de iskaženie [distorsion] comme une réponse aux concepts de sdvig [déplacement ; bougé] et de smeščenie [glissement ; translation] de Tynjanov (p. 212-227). Boris Norman (université d'État de Biélorussie) aperçoit dans la langue actuelle (et surtout dans la publicité) un goût croissant pour la paronomase et les jeux verbaux fondés sur la proximité des signifiants (p. 228-251). Il y voit une riposte créative et réconfortante à l'influence de l'anglo-américain. Citons certains de ces détournements : žest' [tôle] qui prend le sens de "dureté, cruauté », par influence de žestkij [dur], žestokij [cruel]; baton [pain] avec le sens nouveau de "bouton", de l'anglais button; et le savoureux pentjuh [lourdaud], qui désigne un " microprocesseur au pentium ».

6 Les linguistes sont rarement invités dans les prétoires, à la différence des graphologues. C'est à ce sujet original que le célèbre slaviste Daniel Weiss (université de Zurich) consacre une étude passionnante. Il montre que l'instruction judiciaire et le procès sont des lieux où les normes communes de l'interprétation de la langue sont remises en cause. 
Il examine à ce sujet trois types d'implicites: l'absence de marqueurs modaux; la présupposition; les implications conversationnelles (p. 252-274). Les deux derniers articles élargissent le concept de négociation de la norme en observant les médias russes. Lara Rjazanova-Clarke (université d'Edinburgh), qui a cosigné avec Terence Wade The Russian Language Today (1999), analyse le mot vertikal', popularisé par la formule de Vladimir Putin vertikal' vlasti [la verticale du pouvoir] (p. 288-314). Michael S. Gorham (université de Floride) distingue deux types de négociation de la norme : la négociation de type "autoritaire", qui édicte des règles, et celle de type "démocratique», qui établit un dialogue entre les invités et les auditeurs (p. 315-335). Gorham décèle aujourd'hui une approche plus ludique des questions linguistiques. C'est aussi la conclusion des deux éditeurs. Cette attitude globalement plus décontractée n'empêche pas qu'éclatent de temps à autre de furieux débats. Ainsi, en septembre 2009, l'admission du mot kofe [le café (plante et boisson)], normalement masculin, dans le genre neutre souleva un tollé.

On ne peut pas exiger d'un recueil d'articles qu'il ait la cohérence d'une monographie. Les études rassemblées ici présentent naturellement des approches assez différentes et même parfois disparates de la norme et de la " négociation » de celle-ci. La distance est grande, nous l'avons dit, entre le sérieux des expérimentations linguistiques d'un Hlebnikov et l'approche ouvertement ludique de la langue, qu'illustre par exemple aujourd'hui en Russie le phénomène sympathique du «duponisme», du nom de l'écrivain fictif "franco-ouralien» prétendument nommé François Dupont, auteur de trouvailles paronomastiques savoureuses du genre : ničšeagnec [nietzchéagnien, par substitution du mot agnec « agneau » au suffixe <anec>], kul'tja ličnosti [" moignon de la personnalité », par substitution de kul'tja « moignon" à kul't «culte»]; ou de formules détournées: Slova $i$ vešči ne ostavljajte bez vnimanija! [Ne laissez pas vos mots et vos affaires sans surveillance!]. De même, le phénomène des Padonki s'apparente plus à une posture finalement sophistiquée, liée à des phénomènes sociaux contestataires mais marginaux, qu'à un mouvement de fond qui transformerait la langue en profondeur. Après tout, quand il préparait la réforme orthographique de 1917-1918, le grand Dmitrij Ušakov, dans sa correspondance privée, s'amusait, lui aussi, sur le mode plaisant, à déformer l'orthographe des mots. Mais il n'est pas exclu que ces épiphénomènes récents modifient à long ou moyen terme le rapport des russophones à la norme.

Ingunn Lunde et Martin Paulsen apportent avec ce recueil une contribution intéressante à l'étude de la norme langagière dans la Russie d'aujourd'hui. 